\title{
Detection of unreliable measurements in multi-sensor devices
}

\author{
Yednek Asfaw, Andy Adler \\ yasfaw@site.uottawa.ca, Adler@site.uottawa.ca \\ School Of Information Technology and Engineering (SITE) \\ University Of Ottawa, Ottawa, Canada
}

\begin{abstract}
Multi-sensor monitoring devices that use skin surface or implanted sensors are susceptible to changes in temperature, sweat, and movement, such that the measured data cannot be used. This paper presents an automatic approach to detect such erroneous sensors. It is based on the assumption that valid measurements are related by a reconstruction model, while measurements from erroneous sensors are unrelated. The method estimates the data at each sensor based on the measurements from all other sensors, and compares it to the measurements. The sensor-data match is tested using ANOVA to detect the presence of an erroneous sensor. The method was tested on simulated and experimental data of Electrical Impedance Tomography (EIT) and showed consistent identification of erroneous electrodes.
\end{abstract}

Keywords: Data errors, sensors, reconstruction algorithms

\section{INTRODUCTION}

Health care systems worldwide are under pressure to deliver a better and more efficient service. One approach to help deliver such services is technology to enable home and mobile care; several studies have shown that such care improves the patient's quality of life and outcomes, while reducing cost. A key technological component required to enable such care is non-invasive portable biomedical monitoring devices. Such technology enables continuous measurement of parameters as heart and lung activity, blood chemical concentrations, and blood pressure levels.

While a growing market provides great incentive for the researchers and companies in the field, there are difficult challenges associated with design of these devices. The monitoring devices typically use skin surface or implanted sensors to measure the electrical and optical properties of the body. These sensors are susceptible to changes in temperature, sweat, and movement with exercise and breathing. For example, it has been shown that electrodes on the body surface give false readings due to electronic interference [4], patient movement, or sweat and peripheral edema [6], especially in long term monitoring applications [5].

One common technique is the development of heuristic measures to verify the goodness of data. One approach is detection based on identification of unusually large changes in the measurements. Its disadvantage is the difficulty of defining an appropriate threshold for unusual measurements that can be applied across different devices. This paper develops and approach based on explicitly modeling the sensor and device characteristics and using the interrelationships to ascertain the goodness of measurements.

\section{PROPOSED APPROACH}

The paper develops a method to calculate a measure of sensor reliability. It identifies sensors subject to data errors or variability, and calculates a measure of the reliability of each sensor's data. Essentially this work inverses the detection criterion: rather than looking for errors, our approach looks for good data, defined by its consistency with other sensor measurements and prior models of sensor behavior.

\section{System Model}

The system model of a multi-sensor device is expressed as:

$$
\mathbf{z}=\mathbf{H x}
$$

where $\mathbf{H}$ is the linearized observation of model, $\mathbf{z}$ is a vector representation of measurements from all sensors, and $\mathbf{x}$ is the vector of system model parameters.

When the characteristics of the system model are known, the observation model can be determined from the system model, by correlation calculations from test data or from Finite Element Models (FEM). In cases where the system model is unknown, we hypothesize that the observation model may be determined using techniques such as Independent Component Analysis (ICA) [9]. Based on the system model, we calculate a reconstruction model (inverse of the system model) expressed as:

$$
\hat{\mathbf{x}}=\mathbf{R z}
$$

where $\mathbf{R}$ the reconstruction model, and $\hat{\mathbf{x}}$ is the estimate of the system model.

The reconstruction model $(\mathbf{R})$ is determined by inverting the system model equation. If the system model fulfills Hadamard condition [6], then $\mathbf{R}$ can be determined through direct inversion. Otherwise, $\mathbf{R}$ can be determined using a regularization scheme, such as that of [1]. 


\section{Estimation Scheme}

Our method is based on the assumption that a set of good sensors produces internally consistent data. Such consistency can be verified by estimating the measured data at each sensor in the set, using only measurements on other sensors, and then comparing the estimate to the actual data measured. The general procedure for the algorithm is outlined as follows:

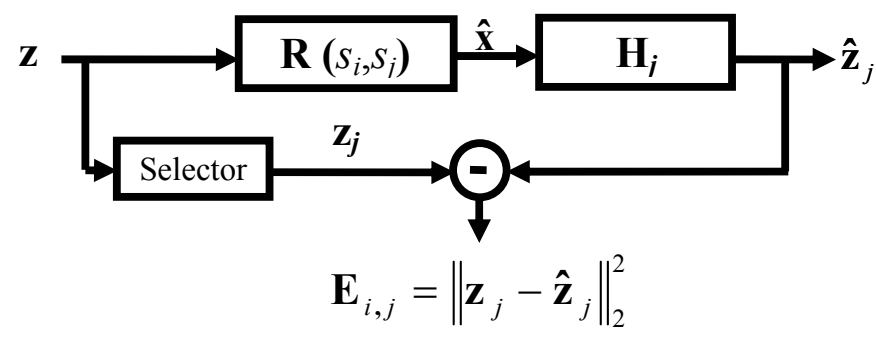

We iterate over each sensor $s_{i}$ in set $A$ (containing all sensors), forming a set $A^{\prime}$ (all sensors not including $s_{i}$ ). $A^{\prime}$ is then tested to calculate a parameter $T_{i}$ which reflects the consistency of measurements among sensors in $A^{\prime}$, and is the sum of estimation errors for all sensors not including $s_{i} . \mathbf{H}_{j}$ represents the rows of the sensitivity matrix $\mathbf{H}$ which correspond to measurements on $s_{j}$. $\hat{\mathbf{x}}$ is then calculated from $\hat{\mathbf{x}}=\mathbf{R}\left(s_{i}, s_{j}\right) \mathbf{z}$, which excludes data from sensors $s_{i}$ and $s_{j}$. The estimate of $\mathbf{z}$ is determined using eq. (1) and the estimation error $\left(\mathbf{E}_{i, j}\right)$ is determined for all possible sensor pairs $s_{i}$ and $s_{j}$. We have shown that $\mathbf{E}_{i, j}$ can be efficiently calculated by precomputing parameters which are independent of data [3].

\section{Decision Parameter}

If all values of $T_{i}$ are low, $A^{\prime}$ contains all "good" sensors, otherwise it contains at least one erroneous sensor. $T_{i}$ values are tested against each other to detect if any are significantly less than the others. This is tested using Analysis of Variance (ANOVA) between $\mathbf{E}_{i}$ of all sensors in set $A$. Using the statistical terminology, $\mathbf{E}_{i}$ are referred to as Treatments [8]. ANOVA is used to determine the statistical similarity between the $\mathbf{E}_{i}$ 's by testing the equality of $\mathrm{N}$ Treatment means $\left(\mu_{1}, \mu_{2}, \mu_{3}, \ldots, \mu_{N}\right)$ [8]. The treatment effect $\left(\tau_{i}\right)$ represents difference of an individual measurement from the overall mean $(\mu)$. The null hypothesis $\left(\mathrm{H}_{0}\right)$ in this case is that no sensors are erroneous, and thus the treatment effect $\left(\tau_{i}\right)$ is equal to zero for all $s_{i}$ in $A$.

$$
\begin{aligned}
& \mathrm{H}_{0}: \tau_{1}=\tau_{2}=\tau_{3}=\ldots=\tau_{N}=0 \\
& \mathrm{H}_{1}: \tau_{i} \neq 0 \text { for at least one } i
\end{aligned}
$$

Under the null hypothesis, each Treatment consists of $\mu$ with a random error component, with observations taken from the normal distribution of $\mu$ with overall variance $\left(\sigma^{2}\right)$. In this case, the estimation error values of $A^{\prime}$ for each candidate sensor $\left(s_{i}\right)$ are similar to one another. The hypothesis is tested by comparing two independent estimates of the population variance:

1. Variance between $\mu_{i}$ and $\mu$ : $\quad\left(\mu_{i}-\mu\right)^{2}$

2. Variance within Treatments: $\left(\mathrm{E}_{i}-\mu_{i}\right)^{2}$

The first variance determines the difference between Treatments and the second variance determines the error within each Treatment. From the above two variances, we calculate sum of squares of Treatments variances:

$$
S S_{T}=\sum_{i=1}^{N}\left(\mu_{i}-\mu\right)^{2}
$$

and sum of squares of error variance:

$$
S S_{E}=\sum_{i=1 j}^{N} \sum_{j=1, j \neq i}^{N}\left(\mathbf{E}_{i, j}-\mu_{i}\right)^{2}
$$

By dividing $S S_{T}$ and $S S_{E}$ by their respective degrees of freedom $(\mathrm{N}-2$ and $((\mathrm{N}-1) \mathrm{N})-1)$, the mean square Treatment $\left(M S_{T}\right)$ and mean square error $\left(M S_{E}\right)$ are calculated. $\mathrm{MS}_{\mathrm{E}}$ is an unbiased estimate of the $\sigma^{2}$ regardless of statistical difference between Treatments. On the other hand, $\mathrm{MS}_{\mathrm{T}}$ is an unbiased estimate of the variance only if $\mathrm{H}_{0}$ is true. The ratio $\mathrm{f}_{0}=\mathrm{MS}_{\mathrm{T}} /$ $\mathrm{MS}_{\mathrm{E}}$ has an $F$-distribution with degree of freedom N-2 and $(\mathrm{N}-1) \mathrm{N}-1$. Hypothesis $\mathrm{H}_{0}$ is rejected if $\mathrm{f}_{0}>\mathrm{f}_{\alpha, \mathrm{N}-2,(\mathrm{~N}-1) \mathrm{N}-1}$ [8]. Thus, using ANOVA, we test if a data set has erroneous sensors, at significance level $\alpha=0.05$.

However, we do not know the location and number of erroneous sensors. To accomplish this task, we use Fisher's Least Significant difference (LSD). LSD compares all pairs of means with the t-statistic. The pairs of means are considered significantly different if

where,

$$
\left|\mu_{i}-\mu_{j}\right|>\operatorname{LSD}
$$

$$
\mathrm{LSD}=\mathrm{t}_{\alpha / 2,(\mathrm{~N}-1) \mathrm{N}-1} \sqrt{\frac{2 M S_{E}}{\mathrm{n}}}
$$

An erroneous sensor will have mean that is significantly different from others $(\mathrm{p}<0.05)$. On the other hand, a "good" sensor will be statistically similar to all other non-erroneous sensors.

\section{RESULTS}

The method was validated using data from Electrical Impedance Tomography (EIT). EIT is an imaging technique which calculates the electrical conductivity distribution within a medium from electrical measurements made at a series of electrodes on the medium surface. EIT is a good candidate for this method because it is a measurement modality in which multiple sets of sensor data are simultaneously acquired. EIT data are acquired by successively applying a low amplitude low frequency current across each pair of electrodes while measuring the voltage 
differences produced on all the other pairs of electrodes. The measurement system used for these experiments has 16 electrodes; for each current pattern, there are 13 electrode pairs not used for current application at which the voltage is read. Data were acquired from a previous study in which mechanically ventilated mongrel dogs were monitored with sixteen EIT electrodes spaced evenly around the shaved thorax [2].

A representative set EIT data of ventilated dogs was used for data with no error (Fig. 1A), and simulated erroneous data from electrode 5 was generated (Fig. 1B). The reconstructed images and graphs of $T$ vs. electrode number are shown. In Fig. 1B, the electrode with errors has significantly lower $T_{i}$ $(p<0.05)$. Fig. 2 shows $\mu_{i}$ vs. electrode number, where the error bars represent the $95 \%$ confidence interval. All the nonerroneous electrodes have a similar confidence interval. However, for the mean of the erroneous electrode, the deviation for the confidence intervals lower and upper limits much is smaller. This suggests the electrode data within $A$, matches better when the erroneous electrode is removed.

Fig $1 \mathrm{~A}$ corresponds to $\mathrm{f}_{0}=1.47$, which is less than $\mathrm{f}_{0.05,14}$, ${ }_{239}=1.67 ; \mathrm{H}_{0}$ cannot be reject at $\mathrm{p}<.05$, and we conclude there are no erroneous electrodes. On the other hand, the null hypothesis is rejected for Fig $1 \mathrm{~B}$ since $\mathrm{f}_{0}=2.71$ is significantly higher than $\mathrm{f}_{0.05,14,239}$.

To determine the sensitivity of the method, white Gaussian noise was added to the data of a particular electrode from a representative clean data. Data were calculated for SNR values from $-50 \mathrm{~dB}$ to $50 \mathrm{~dB}$, where simulations are repeated 100 times to calculate the error distribution. The resulting $\mathrm{F}$ ratio vs. SNR graph indicates the method can reliably detect an erroneous electrode when the SNR is below approximately $5 \mathrm{~dB}$.

\section{CONCLUSION}

In this paper, we have presented a method to automatically detect erroneous sensors in multi-sensor system, such as EIT. The method is based on the model that an erroneous sensor produces measurements inconsistent with those from other good sensors. Results show that the method is able to correctly detect the presence of and identify the location of erroneous sensors in representative EIT data.

The decision criterion is based on ANOVA and shows that detection of one or more erroneous sensor is feasible for SNR less than $5 \mathrm{~dB}$ with $\mathrm{p}<0.05$. Automatic detection of sensor errors in multi-sensor system has several possible applications. In offline processing, such a technique could identify and correct for such errors. More usefully, if implemented in multi-sensor monitoring equipment, it would be possible to alert staff who could then attend to the problem. However, for such online applications, the algorithm is still slow (5s) for real-time data analysis, but would permit erroneous sensor detection in the background.
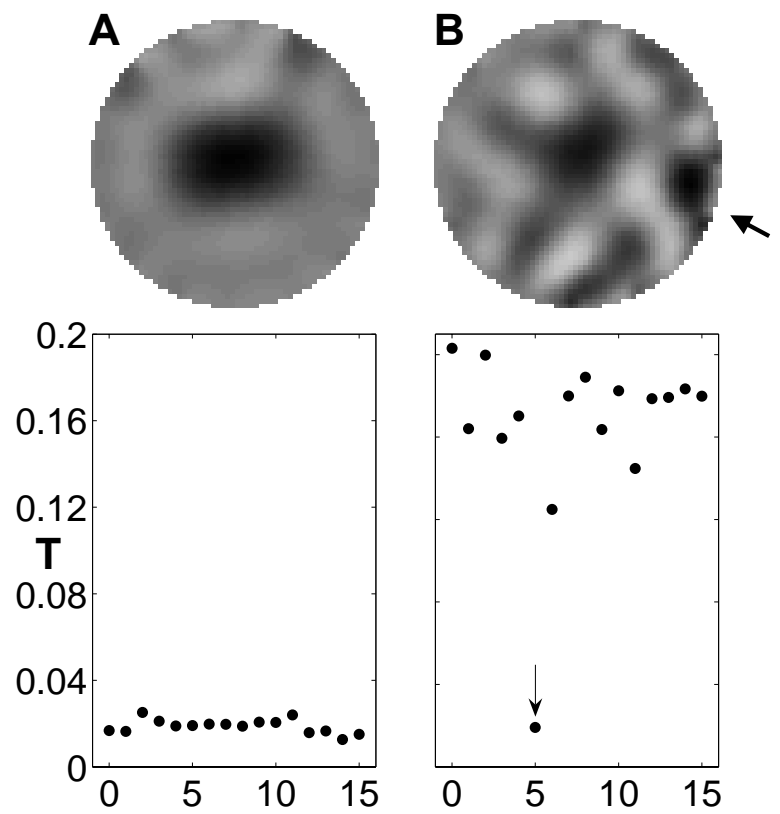

Figure 1: Top: (A) Reconstructed conductivity distribution of a dog thorax with no electrode error. (B) Reconstructed conductivity distribution of dog thorax with simulated erroneous electrode data of $\mathrm{SNR}=-10 \mathrm{~dB}$ to electrode 5 . Electrodes are numbered in the clockwise direction starting at 12 o'clock position. Dark colors are regions of low conductivity. Bottom: (A) $T$ vs. electrode graph shows consistency in the absence of no erroneous electrode. (B) $T$ vs. electrode graph shows $T$ for electrode 5 is much lower than the rest of the $T$ values, suggesting that electrode 5 is erroneous

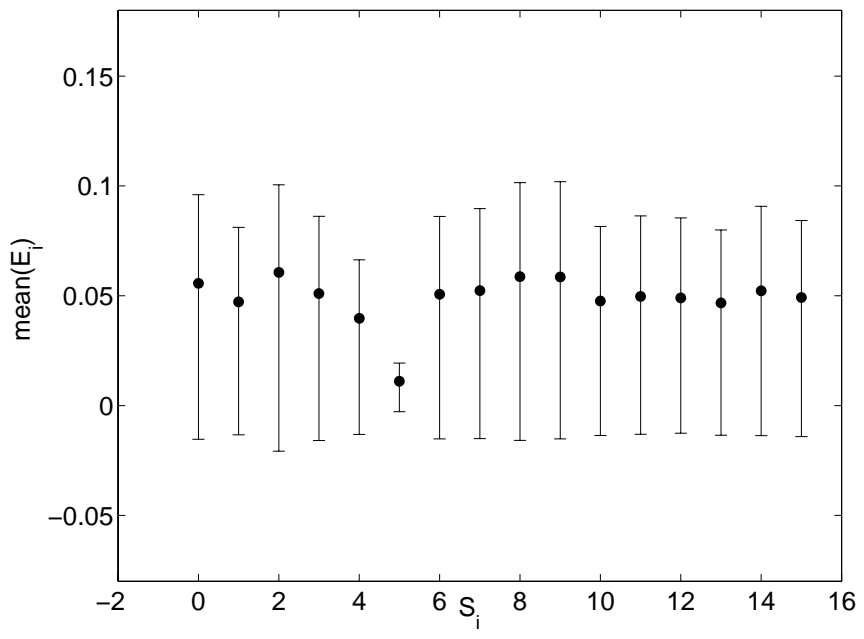

Figure 2: The mean values of $\mathbf{E}_{i}$ for all electrodes in $A$ of Fig. 1B. The erroneous electrode has a smaller $\mu$ with smaller difference between the limits of the confidence interval as compared to the "good" electrodes. 


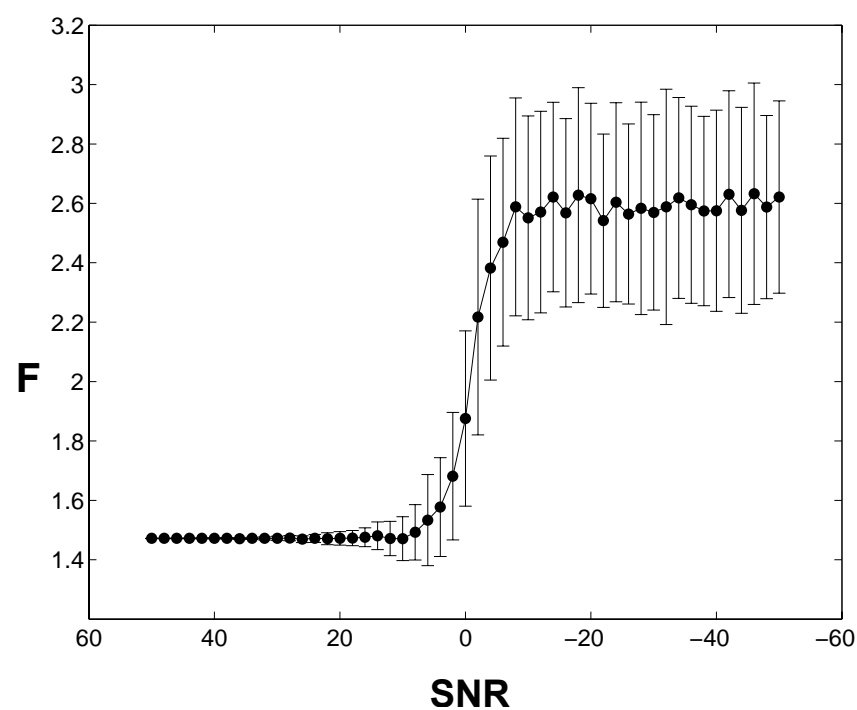

Figure 3: F statistic ( \pm Std Dev) vs. SNR: Representative "good" data were used to analyze the sensitivity of the method. White Gaussian noise was added to electrode 5 (SNR: $-50 \mathrm{~dB}$ to $50 \mathrm{~dB}$ ). The experiment was repeated to determine the margin of error. The threshold for detection is at a SNR of $5 \mathrm{~dB}$.

\section{REFERENCES}

[1] A Adler and $\mathrm{R}$ Guardo, "Electrical Impedance Tomography: Regularised imaging and Contrast Detection", IEEE Trans. Medical Imag. Vol. 15, No. 2, pp. 170-179, April 1996

[2] A Adler, R Amyot, R Guardo, J H T Bates, Y Berthiaume, "Monitoring changes in lung air and liquid volumes with electrical impedance tomography", J. Appl. Physiol., Vol. 83, No. 5, pp. 1762-1767, Nov 1997

[3] Y Asfaw and A Adler, "Automatic detection of erroneous electrodes in Electrical Impedance Tomography”, Physiol. Meas., In press

[4] F Al-Hatib, "Patient-instrument connection errors in bioelectrical impedance measurement", Physiol. Meas., Vol. 19, No. 2, pp. 285-296, May 1998

[5] A Lozano, J Rosell and R Pallás-Areny, "Errors in prolonged electrical impedance measurements due to electrode repositioning and postural changes", Physiol. Meas., Vol. 16, No. 2, pp. 121-130, May 1995

[6] S Meeson, B Blott, and A Killingback, "EIT data noise evaluation in the clinical environment", Physiol. Meas., Vol. 17(suppl.), No. 4A, pp. A33-A38, November 1996

[7] R Aster, B Borchers, and C Thurber, "Parameter Estimation and Inverse Problems", Academic Press, 2004

[8] DC Montgomery and GC Runger, "Applied Statistics and Probability for Engineers Chapter 13: Design and analysis of single factor experiments: The analysis of variance", $3^{\text {rd }}$ ed, John Wiley \& sons inc, 2002

[9] CJ James and CW Hesse, "Independent component analysis for biomedical signals", Physiol. Meas., Vol. 26 , No. 1, pp. R15-R39, February 2005 